\title{
Paleoenvironment of the Lower Ordovician Meitan Formation in the Sichuan Basin and Adjacent Areas, China
}

\author{
Meihua Yang ${ }^{1}$, Yinhui Zuo ${ }^{1, *(\mathbb{D}}$, Xiaodong $\mathrm{Fu}^{2}$, Lei Qiu ${ }^{1,3}$, Wenzheng $\mathrm{Li}^{2}$, Jianyong Zhang ${ }^{2}$, Ziyun Zheng ${ }^{1}$ \\ and Jiazhen Zhang ${ }^{1}$
}

check for

updates

Citation: Yang, M.; Zuo, Y.; Fu, X.; Qiu, L.; Li, W.; Zhang, J.; Zheng, Z.; Zhang, J. Paleoenvironment of the Lower Ordovician Meitan Formation in the Sichuan Basin and Adjacent Areas, China. Minerals 2022, 12, 75. https://doi.org/10.3390/min12010075

Academic Editors: Paula

Alexandra Gonçalves and

Carolina Fonseca

Received: 18 November 2021

Accepted: 5 January 2022

Published: 8 January 2022

Publisher's Note: MDPI stays neutral with regard to jurisdictional claims in published maps and institutional affiliations.

Copyright: () 2022 by the authors. Licensee MDPI, Basel, Switzerland. This article is an open access article distributed under the terms and conditions of the Creative Commons Attribution (CC BY) license (https:// creativecommons.org/licenses/by/ $4.0 /)$.
1 State Key Laboratory of Oil and Gas Reservoir Geology and Exploitation, Chengdu University of Technology, Chengdu 610059, China; yang_mh0616@126.com (M.Y.); qiulei.xnyq@sinopec.com (L.Q.); yun1406808626@126.com (Z.Z.); zhangjiazhen2021@126.com (J.Z.)

2 PetroChina Hangzhou Research Institute of Geology, Hangzhou 310023, China; fuxd_hz@petrochina.com.cn (X.F.); liwz_hz@petrochina.com.cn (W.L.); zhangjy_hz@petrochina.com.cn (J.Z.)

3 4th Producting Plant, Southwest Oil \& Gas Company, SINOPEC, Chongqing 402160, China

* Correspondence: zuoyinhui@tom.com; Tel.: +86-84079008

\begin{abstract}
The quality of hydrocarbon source rocks is affected by the sedimentary paleoenvironment. A paleoenvironment with anoxia and a high paleoproductivity is beneficial to source rocks. The paleoenvironment of the Lower Ordovician Meitan Formation of the Sichuan Basin and its adjacent areas is lacking, restricting the oil and gas exploration of the Ordovician in the Sichuan Basin and its adjacent areas. In this paper, the content of major and trace elements of 50 samples was tested to clarify the paleoenvironment of the Meitan Formation. The paleoclimate, paleosalinity, paleoredox, and paleoproductivity during the deposition of the Meitan Formation were analyzed. The control effect of the paleoenvironment on the development of source rocks was clarified, and the favorable paleoenvironment for source rock development was pointed out. The results show that the paleoenvironment of the Meitan Formation has the following characteristics: humidity, brackish water, oxygen depletion, anoxia environment, and high paleoproductivity. These characteristics are conducive to the development of poor and moderate source rocks. The source rocks of the Meitan Formation were developed in the north, west, and south of the Sichuan Basin and its adjacent areas. The organic matter of the source rocks is mainly typed $\mathrm{II}_{1}$ kerogen, and the quality is evaluated as poor-medium source rocks having the potential of generating oil and gas. This study can provide fundamental parameters for the further exploration of Ordovician petroleum.
\end{abstract}

Keywords: paleoenvironment; mudstone; source rock; Meitan Formation; Sichuan Basin and its adjacent areas

\section{Introduction}

The hydrocarbon generation and the expulsion intensity and amount are not only affected by tectonic thermal evolutions, but also by the quality of source rocks, including organic matter abundance, type, and maturity. These parameters are affected by the paleoclimate and sedimentary environment, such as paleosalinity, paleoredox, and paleoproductivity [1-3].

The Ordovian of the Sichuan Basin and its adjacent areas, in southwestern China (Figure 1), have been regarded as a concurrent layer for oil and gas exploration of the Silurian and the Cambrian, and no large oil and gas fields have been found. Moreover, from the Sinian to the Jurassic, the Ordovician is the only exploration strata that have not been commercially discovered [4]. At present, the oil and gas discoveries of the Ordovician in the Sichuan Basin and its adjacent areas are mainly concentrated in the Lower Ordovician Tongzi Formation and the Upper Ordovician Baota Formation, and the karst-fractured reservoirs are mainly found [5]. 


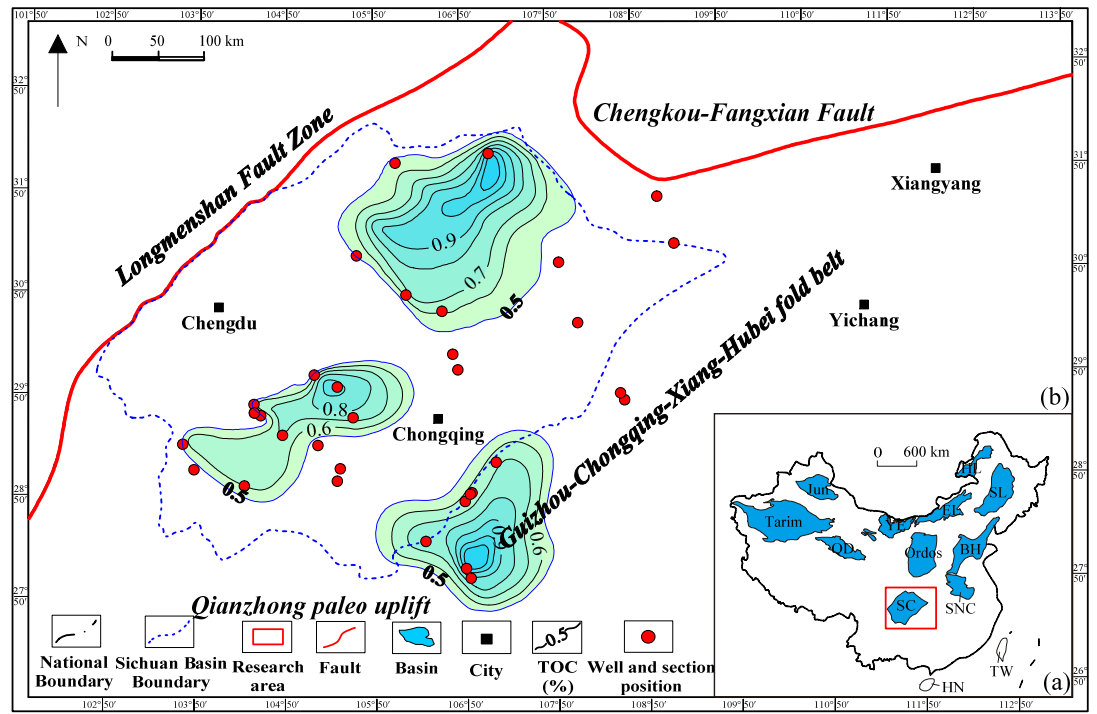

Figure 1. (a) Petroliferous basins of China and position of the Sichuan Basin and its adjacent areas; (b) Structural division and TOC of the Sichuan Basin and its adjacent areas. TOC = Total organic carbon; Jun = Junnggar Basin; YE = Yingen-Ejinaqi Basin; EL = Erlian Basin; HL = Hailaer Basin; $\mathrm{SL}=$ Songliao Basin; $\mathrm{QD}=$ Qaidam Basin; $\mathrm{BH}=$ Bohai Bay Basin; $\mathrm{SC}=$ Sichuan Basin.

Previous studies have shown that the Lower Ordovician Meitan Formation has the most important source rock of the Ordovician in the Sichuan Basin and its adjacent areas $[6,7]$. However, there are few basic data for the study of the source rock of the Meitan Formation, only a small amount of outcrop sections and drilling data, and a lack of basic parameters such as seismic data and organic geochemical analyses. It is impossible to systematically analyze the paleoenvironment and the influences on source rocks, which seriously limits the oil and gas exploration of the Ordovician.

In this paper, the paleoclimate, paleosalinity, paleoredox, and paleoproductivity of the Meitan Formation were analyzed in combination with geological and geochemical parameters. This paper reveals the control of the paleoenvironment on the development of the source rocks and pointed out the favorable paleoenvironment of the source rock. It is expected to provide theoretical support for the assessment of the resource potential and exploration target optimization of the Meitan Formation.

\section{Geological Settings}

The Sichuan Basin and its adjacent areas are located in southwestern China (Figure 1a). The Songpan-Ganzi fold belt and the Longmenshan fault zone are in the west of the research area, the Chengkou-Fangxian fault is in the north, the Guizhou-Chongqing-XiangHubei fold belt is in the east, and the Qianzhong paleo uplift is in the south. The Sichuan Basin is distributed in the study area in a NE direction, showing the characteristics of a multi-tectonic system under multi-stage structures (Figure 1b) [8].

From the Late Cambrian to the Early Silurian, several strata developed in the Sichuan Basin and its adjacent areas. The Lower Ordovician Tongzi Formation, Honghuayuan Formation, Meitan Formation, Middle Ordovician Shizipu Formation, Upper Ordovician Baota Formation, Linxiang Formation, and Wufeng Formation were deposited from the bottom to the top (Figure 2). However, the Middle-Upper Ordovician were missing in the north, west, and southwest of the Sichuan Basin under the influence of the Caledonian movement $[6,9]$. 


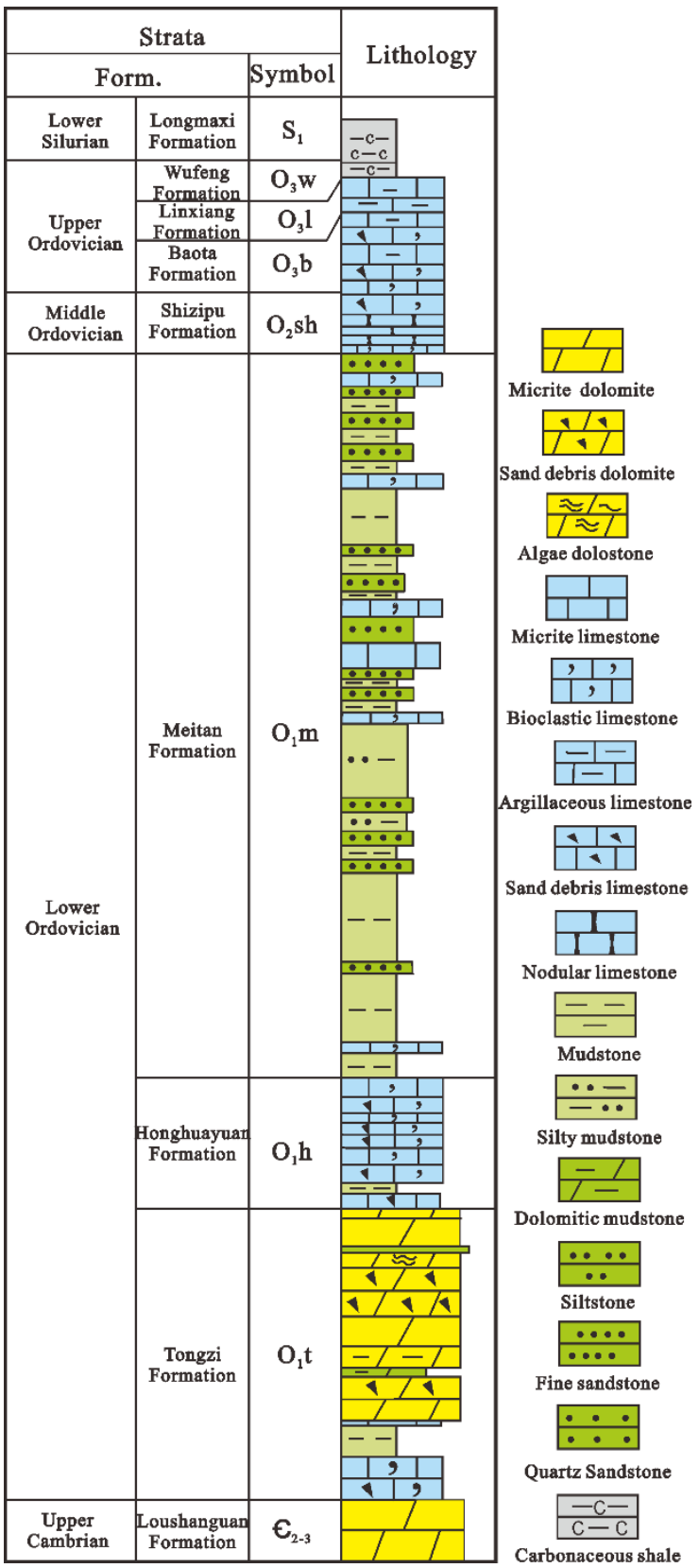

Figure 2. Stratigraphic column map of the Ordovician of the Sichuan Basin and its adjacent areas.

The Meitan Formation's depositional period was affected by multiple sea-level fluctuations. During the early depositional period of the Meitan Formation, the basement rapidly subsided, and the sea level was rising under the affection of the Caledonian movement. The depositional characteristics were characterized by shallow cement shelf facies dominated by mudstones. During the middle Meitan Formation deposit, the sea level dropped, and the terrigenous debris was deposited. The lithology is characterized by siltstone, fine sandstone, and limestone, mainly through mixed shelf facies deposition. During the late depositional period of the Meitan Formation, the sea level rose again, marked by the end of deep-water shelf facies deposition, and limestone, bioclastic limestone, and sandy limestone were mainly developed [10,11].

\section{Materials and Methods}

In this paper, typical wells and geological sections of the Sichuan Basin and its adjacent areas were selected for geochemical experiments. The major and trace element data were 
taken from 10 mudstone samples from 2 wells and 42 mudstone samples from 4 geological sections (Tables 1 and 2). Total organic carbon (TOC) data were taken from 486 mudstone samples from 23 wells and 7 geological sections, and kerogen carbon isotope data were taken from 20 mudstone samples from 3 wells and 2 geological sections.

Table 1. Major elements of mudstones in the Meitan Formation.

\begin{tabular}{|c|c|c|c|c|c|c|c|c|c|c|c|}
\hline $\begin{array}{c}\text { Well and } \\
\text { Section No. }\end{array}$ & Lithology & $\begin{array}{l}\mathrm{Na} \\
(\%)\end{array}$ & $\begin{array}{l}\mathrm{Mg} \\
(\%)\end{array}$ & $\begin{array}{c}\text { Al } \\
(\%)\end{array}$ & $\begin{array}{c}\mathrm{Si} \\
(\%)\end{array}$ & $\begin{array}{l}\text { Mn } \\
(\%)\end{array}$ & $\begin{array}{c}K \\
(\%)\end{array}$ & $\begin{array}{l}\mathrm{Ca} \\
(\%)\end{array}$ & $\begin{array}{c}\mathrm{Ti} \\
(\%)\end{array}$ & $\begin{array}{c}P \\
(\%)\end{array}$ & $\begin{array}{c}\mathrm{Fe} \\
(\%)\end{array}$ \\
\hline JT1 & Gray calcareous mudstone & 0.28 & 1.18 & 6.99 & 16.48 & 0.05 & 3.68 & 14.46 & 0.28 & 0.05 & 3.68 \\
\hline JT1 & Gray calcareous mudstone & 0.34 & 1.01 & 5.78 & 14.93 & 0.03 & 2.94 & 17.95 & 0.24 & 0.05 & 2.93 \\
\hline JT1 & Gray calcareous mudstone & 0.36 & 0.97 & 7.83 & 19.3 & 0.02 & 4 & 9.25 & 0.28 & 0.06 & 4.62 \\
\hline JT1 & Gray calcareous mudstone & 0.43 & 1.34 & 9.12 & 23.26 & 0.08 & 4.59 & 4.18 & 0.42 & 0.1 & 4.68 \\
\hline JT1 & Gray calcareous mudstone & 1.37 & 0.3 & 10.66 & 22.53 & 0.02 & 4.6 & 4.26 & 0.33 & 0.06 & 4.42 \\
\hline WK1 & Grayish black mudstone & 0.73 & 1.54 & 10.84 & 24.94 & 0.06 & 4.29 & 0.93 & 0.43 & 0.06 & 5.96 \\
\hline WK1 & Deep gray mudstone & 0.67 & 1.58 & 11.33 & 25.16 & 0.05 & 4.39 & 0.56 & 0.44 & 0.06 & 5.75 \\
\hline WK1 & $\begin{array}{l}\text { Deep gray sandy } \\
\text { mudstone }\end{array}$ & 0.63 & 1.62 & 11.41 & 25.06 & 0.05 & 4.17 & 0.51 & 0.45 & 0.05 & 6.27 \\
\hline WK1 & Deep gray mudstone & 0.65 & 1.58 & 11.35 & 25.26 & 0.05 & 4.20 & 0.49 & 0.44 & 0.06 & 6.00 \\
\hline WK1 & Grayish black mudstone & 0.72 & 1.57 & 10.68 & 25.61 & 0.05 & 3.89 & 0.87 & 0.42 & 0.07 & 6.08 \\
\hline QJ & Deep gray mudstone & 0.67 & 1.66 & 9.06 & 25.38 & 0.50 & 3.52 & 2.35 & 0.45 & 0.11 & 4.96 \\
\hline QJ & Black mudstone & 0.63 & 1.61 & 9.39 & 25.08 & 0.50 & 3.70 & 2.15 & 0.46 & 0.11 & 5.07 \\
\hline QJ & Shallow black mudstone & 0.64 & 1.39 & 10.18 & 25.86 & 0.32 & 3.87 & 0.60 & 0.51 & 0.13 & 5.30 \\
\hline QJ & Dark black mudstone & 0.41 & 1.43 & 11.39 & 24.40 & 0.07 & 4.52 & 0.40 & 0.45 & 0.05 & 5.40 \\
\hline $\mathrm{QJ}$ & Deep gray mudstone & 0.46 & 1.36 & 11.00 & 25.54 & 0.08 & 4.35 & 0.49 & 0.48 & 0.07 & 4.73 \\
\hline QJ & Deep gray mudstone & 0.48 & 1.38 & 11.56 & 25.13 & 0.06 & 4.63 & 0.48 & 0.54 & 0.07 & 4.23 \\
\hline QJ & Deep gray mudstone & 0.71 & 1.26 & 10.79 & 26.24 & 0.04 & 4.49 & 0.51 & 0.50 & 0.10 & 3.98 \\
\hline QJ & Grayish black mudstone & 0.92 & 1.37 & 9.85 & 27.04 & 0.10 & 4.05 & 0.64 & 0.59 & 0.14 & 4.62 \\
\hline QJ & Grayish black mudstone & 0.43 & 1.46 & 11.80 & 24.41 & 0.05 & 5.28 & 0.41 & 0.47 & 0.06 & 4.43 \\
\hline WXT & Dark black mudstone & 0.56 & 1.62 & 8.73 & 25.73 & 0.03 & 3.29 & 3.17 & 0.44 & 0.05 & 4.06 \\
\hline WXT & Dark black mudstone & 0.56 & 1.61 & 8.74 & 25.57 & 0.02 & 3.30 & 3.04 & 0.44 & 0.05 & 4.20 \\
\hline WXT & Dark black mudstone & 0.57 & 1.63 & 8.66 & 25.27 & 0.03 & 3.22 & 3.54 & 0.44 & 0.05 & 4.32 \\
\hline WXT & Dark black mudstone & 0.48 & 1.70 & 8.65 & 24.25 & 0.03 & 3.34 & 4.52 & 0.44 & 0.05 & 4.14 \\
\hline WXT & Dark black mudstone & 0.42 & 1.84 & 9.42 & 25.59 & 0.02 & 3.66 & 1.81 & 0.43 & 0.04 & 4.41 \\
\hline WXT & Dark black mudstone & 0.37 & 1.84 & 9.47 & 25.76 & 0.03 & 3.60 & 1.04 & 0.44 & 0.04 & 4.51 \\
\hline WXT & Dark black mudstone & 0.41 & 1.71 & 8.16 & 23.17 & 0.04 & 3.14 & 6.28 & 0.39 & 0.04 & 3.92 \\
\hline WXT & Dark black mudstone & 0.40 & 1.75 & 8.89 & 24.38 & 0.03 & 3.46 & 4.04 & 0.39 & 0.04 & 3.95 \\
\hline WXT & Dark black mudstone & 0.39 & 1.76 & 8.95 & 24.44 & 0.03 & 3.50 & 4.01 & 0.41 & 0.04 & 4.11 \\
\hline WXT & Dark black mudstone & 0.43 & 1.78 & 9.06 & 24.67 & 0.03 & 3.46 & 3.09 & 0.42 & 0.04 & 4.18 \\
\hline PD & Yellow-green mudstone & 0.34 & 1.22 & 10.51 & 24.71 & 0.02 & 3.59 & 0.38 & 0.43 & 0.06 & 6.08 \\
\hline PD & Yellow-green mudstone & 0.38 & 1.34 & 11.44 & 24.44 & 0.01 & 4.28 & 0.40 & 0.46 & 0.05 & 5.95 \\
\hline PD & Gray-green mudstone & 0.23 & 1.22 & 11.48 & 24.34 & 0.04 & 4.62 & 0.47 & 0.46 & 0.06 & 5.61 \\
\hline PD & Yellow-green mudstone & 0.32 & 1.26 & 11.56 & 24.75 & 0.05 & 4.51 & 0.51 & 0.48 & 0.05 & 4.70 \\
\hline $\mathrm{BQ}$ & Deep gray mudstone & 0.24 & 1.42 & 11.51 & 25.57 & 0.01 & 4.89 & 0.26 & 0.46 & 0.06 & 6.11 \\
\hline $\mathrm{BQ}$ & Deep gray mudstone & 0.16 & 1.38 & 11.34 & 25.8 & 0.02 & 4.66 & 0.08 & 0.48 & 0.06 & 6.16 \\
\hline $\mathrm{BQ}$ & Deep gray mudstone & 0.6 & 1.68 & 10.81 & 26.08 & 0.03 & 4.24 & 0.78 & 0.47 & 0.08 & 5.47 \\
\hline$\hat{B Q}$ & Deep gray mudstone & 1.56 & 0.79 & 6.1 & 23.47 & 0.08 & 2.14 & 11.17 & 0.36 & 0.06 & 2.36 \\
\hline $\mathrm{BQ}$ & Deep gray mudstone & 1.23 & 1.63 & 11 & 27.32 & 0.01 & 4.57 & 0.47 & 0.53 & 0.09 & 4.01 \\
\hline $\mathrm{BQ}$ & Deep gray mudstone & 0.73 & 2.18 & 11.12 & 24.39 & 0.02 & 4.62 & 0.34 & 0.5 & 0.06 & 7.14 \\
\hline$\hat{\mathrm{BQ}}$ & Deep gray mudstone & 0.44 & 2.16 & 12.4 & 23.59 & 0.02 & 5.67 & 0.29 & 0.5 & 0.05 & 5.71 \\
\hline BQ & Deep gray mudstone & 1.57 & 0.92 & 7.09 & 27.65 & 0.07 & 2.79 & 5.57 & 0.32 & 0.12 & 3.12 \\
\hline BQ & Deep gray mudstone & 0.81 & 1.66 & 10.56 & 25.83 & 0.02 & 4.86 & 0.5 & 0.44 & 0.08 & 5.91 \\
\hline BQ & Deep gray mudstone & 0.83 & 1.6 & 9.86 & 25.8 & 0.08 & 4.42 & 2.01 & 0.48 & 0.09 & 5.07 \\
\hline BQ & Deep gray mudstone & 0.52 & 0.61 & 2.83 & 10.96 & 0.23 & 1.16 & 24.33 & 0.14 & 0.76 & 4.03 \\
\hline BQ & Deep gray mudstone & 0.82 & 1.81 & 9.81 & 27.38 & 0.02 & 4.59 & 0.67 & 0.52 & 0.08 & 4.63 \\
\hline $\mathrm{BQ}$ & Deep gray mudstone & 1.24 & 1.02 & 7.6 & 28.49 & 0.06 & 3.86 & 4 & 0.56 & 0.21 & 2.89 \\
\hline $\mathrm{BQ}$ & Deep gray mudstone & 1.1 & 1.58 & 9.61 & 27.7 & 0.02 & 4.59 & 0.78 & 0.61 & 0.22 & 4.18 \\
\hline BQ & Deep gray mudstone & 0.94 & 1.65 & 9.35 & 26.58 & 0.04 & 4.53 & 2.03 & 0.59 & 0.24 & 3.91 \\
\hline$\hat{\mathrm{BQ}}$ & Deep gray mudstone & 1.18 & 1.38 & 7.65 & 26.55 & 0.09 & 3.56 & 4.51 & 0.62 & 0.32 & 3.85 \\
\hline $\mathrm{BQ}$ & Deep gray mudstone & 0.5 & 0.8 & 3.64 & 14.94 & 0.08 & 1.6 & 21.98 & 0.19 & 0.03 & 2.02 \\
\hline
\end{tabular}


Table 2. Trace elements of mudstones in the Meitan Formation.

\begin{tabular}{|c|c|c|c|c|c|c|c|c|c|c|c|c|c|c|c|c|}
\hline \multirow{2}{*}{ Well and Section No. } & \multirow{2}{*}{ Lithology } & $\mathbf{L i}$ & Be & $\mathbf{V}$ & Co & $\mathbf{N i}$ & $\mathrm{Cu}$ & Zn & $\mathbf{R b}$ & Sr & Mo & $\mathrm{Ba}$ & $\mathbf{P b}$ & Th & $\mathbf{U}$ & $\mathrm{Cr}$ \\
\hline & & \multicolumn{15}{|c|}{ ppm } \\
\hline JT1 & Gray calcareous mudstone & 36.9 & 2.24 & 93 & 13.8 & 33.2 & 30.2 & 53.2 & 162 & 460 & 1.56 & 5908 & 17.7 & 15.4 & 3.44 & 102 \\
\hline JT1 & Gray calcareous mudstone & 27.2 & 1.65 & 73.7 & 10.7 & 25.3 & 24.9 & 52.9 & 123 & 506 & 3.65 & 4909 & 15.5 & 13.5 & 3.91 & 100 \\
\hline JT1 & Gray calcareous mudstone & 41.9 & 2.31 & 108 & 15.2 & 42.7 & 37.3 & 70.7 & 173 & 451 & 3.26 & 12945 & 31.8 & 16.5 & 3.28 & 126 \\
\hline JT1 & Gray calcareous mudstone & 44.8 & 2.92 & 114 & 17.4 & 41.4 & 33.1 & 105 & 194 & 290 & 2.36 & 6411 & 30.4 & 20 & 3.43 & 129 \\
\hline JT1 & Gray calcareous mudstone & 35.6 & 3.04 & 129 & 17.9 & 47.6 & 48 & 55.9 & 173 & 312 & 9.26 & 13411 & 40.3 & 19.7 & 3.81 & 155 \\
\hline WK1 & Grayish black mudstone & 66.6 & 4.35 & 127 & 27 & 46.4 & 34.5 & 81 & 220 & 121.5 & 0.42 & 970 & 22 & 22.3 & 2.69 & 130 \\
\hline WK1 & Deep gray mudstone & 71.7 & 4.13 & 143 & 19 & 47.6 & 40.7 & 103 & 219 & 101.5 & 0.37 & 971 & 30.4 & 20.2 & 2.65 & 180 \\
\hline WK1 & Deep gray mudstone & 81.9 & 4.17 & 147 & 17.9 & 48 & 41.6 & 113 & 209 & 93.3 & 0.21 & 933 & 10.7 & 20.3 & 2.53 & 140 \\
\hline WK1 & Grayish black mudstone & 77.5 & 3.92 & 130 & 23.5 & 46.5 & 36.2 & 107 & 193 & 146.5 & 0.36 & 895 & 15.1 & 21 & 2.61 & 130 \\
\hline QJ & Deep gray mudstone & 55.6 & 4.51 & 111 & 18.6 & 43.3 & 34.3 & 115 & 206 & 157 & 1.28 & 631 & 19 & 17.6 & 2.62 & 97.6 \\
\hline QJ & Black mudstone & 54.6 & 4.43 & 116 & 18.2 & 40.4 & 31.7 & 117 & 215 & 127 & 1.05 & 652 & 25.7 & 19.5 & 2.64 & 103 \\
\hline QJ & Shallow black mudstone & 53.6 & 4.3 & 123 & 18.7 & 45 & 29.5 & 94.9 & 209 & 102 & 1.45 & 1017 & 18.9 & 18 & 2.45 & 115 \\
\hline QJ & Dark black mudstone & 49.9 & 4.16 & 134 & 15.3 & 45.4 & 39.3 & 83 & 249 & 94.2 & 0.9 & 854 & 19.9 & 18.8 & 2.51 & 106 \\
\hline QJ & Deep gray mudstone & 47 & 4.33 & 130 & 17.5 & 48.4 & 38 & 71.4 & 241 & 101 & 0.91 & 906 & 19 & 18.8 & 2.45 & 113 \\
\hline QJ & Deep gray mudstone & 39.8 & 4.65 & 146 & 12.8 & 44.2 & 35.8 & 75.9 & 239 & 88.6 & 0.98 & 1109 & 12.5 & 20.2 & 2.64 & 130 \\
\hline QJ & Grayish black mudstone & 41.4 & 4.31 & 147 & 12.3 & 48.6 & 47.7 & 84.8 & 252 & 73.1 & 1.76 & 1392 & 12.7 & 18.6 & 2.58 & 110 \\
\hline WXT & Dark black mudstone & 40.4 & 2.74 & 150 & 15.6 & 42.5 & 42.6 & 95.3 & 209 & 147 & 3.42 & 793 & 27.5 & 18.8 & 3.3 & 92.9 \\
\hline WXT & Dark black mudstone & 41.4 & 2.69 & 155 & 13.2 & 47.2 & 46.1 & 100 & 216 & 147 & 3.86 & 782 & 28.2 & 18.3 & 3.48 & 90.6 \\
\hline WXT & Dark black mudstone & 41.6 & 2.82 & 147 & 18.4 & 49.9 & 45.4 & 103 & 210 & 167 & 4.56 & 783 & 28.1 & 18.8 & 3.61 & 97.6 \\
\hline WXT & Dark black mudstone & 40.4 & 2.71 & 132 & 16.1 & 39.5 & 36.7 & 92.5 & 206 & 158 & 3 & 790 & 26.7 & 17.5 & 3.5 & 83 \\
\hline WXT & Dark black mudstone & 46.9 & 3.03 & 181 & 14.1 & 53.6 & 43.7 & 120 & 236 & 96.1 & 2.11 & 992 & 20.1 & 17.7 & 3.39 & 92.6 \\
\hline WXT & Dark black mudstone & 47.1 & 3.15 & 204 & 14.3 & 56.9 & 44.1 & 129 & 234 & 92 & 1.8 & 886 & 26.7 & 18.8 & 3.93 & 104 \\
\hline WXT & Dark black mudstone & 39.6 & 2.47 & 131 & 15.6 & 47.5 & 37.5 & 105 & 207 & 231 & 3.07 & 1103 & 20.8 & 14.4 & 3.51 & 82.1 \\
\hline WXT & Dark black mudstone & 40 & 2.52 & 144 & 14.1 & 44.8 & 35.2 & 101 & 218 & 134 & 2.8 & 989 & 21.6 & 16.3 & 3.49 & 90.1 \\
\hline WXT & Dark black mudstone & 44.3 & 2.78 & 162 & 17 & 47.3 & 37.4 & 112 & 234 & 155 & 2.73 & 1191 & 23.7 & 16.2 & 3.33 & 91 \\
\hline WXT & Dark black mudstone & 43.5 & 2.65 & 135 & 16 & 43.2 & 33.4 & 108 & 240 & 152 & 1.37 & 2236 & 19 & 16.6 & 3.15 & 86.8 \\
\hline PD & Yellow-green mudstone & 53.2 & 2.92 & 130 & 14.6 & 37.7 & 50.1 & 61.2 & 215 & 67.2 & 0.84 & 380 & 8.14 & 12.8 & 1.58 & 90 \\
\hline
\end{tabular}


Table 2. Cont.

\begin{tabular}{|c|c|c|c|c|c|c|c|c|c|c|c|c|c|c|c|c|}
\hline \multirow{2}{*}{ Well and Section No. } & \multirow{2}{*}{ Lithology } & $\mathbf{L i}$ & Be & $\mathbf{V}$ & Co & $\mathrm{Ni}$ & $\mathrm{Cu}$ & $\mathrm{Zn}$ & $\mathbf{R b}$ & Sr & Mo & $\mathbf{B a}$ & $\mathbf{P b}$ & Th & $\mathbf{U}$ & $\mathrm{Cr}$ \\
\hline & & \multicolumn{15}{|c|}{ ppm } \\
\hline PD & Yellow-green mudstone & 39.4 & 2.46 & 131 & 15 & 37.7 & 30.5 & 65.4 & 213 & 68.4 & 0.84 & 356 & 9.17 & 12.9 & 1.53 & 94.1 \\
\hline PD & Gray-green mudstone & 43.9 & 4.16 & 131 & 26.9 & 49.6 & 39.4 & 124 & 188 & 95.8 & 0.87 & 658 & 17.1 & 14.4 & 1.66 & 115 \\
\hline PD & Yellow-green mudstone & 47 & 3.27 & 131 & 14.9 & 42.4 & 28.4 & 98.1 & 236 & 109 & 0.46 & 645 & 10 & 15 & 1.74 & 111 \\
\hline PD & Deep gray mudstone & 39.5 & 3.54 & 142 & 19.6 & 42.2 & 42.7 & 99.4 & 267 & 91.4 & $<0.20$ & 704 & 5.9 & 16.8 & 1.69 & 120 \\
\hline PD & Deep gray mudstone & 39.2 & 3.55 & 155 & 15.4 & 35.2 & 38.9 & 94.9 & 247 & 93.6 & 0.45 & 670 & 13.6 & 17.3 & 2.22 & 120 \\
\hline $\mathrm{BQ}$ & Deep gray mudstone & 36.2 & 4.37 & 125 & 13.5 & 44.8 & 27.1 & 91.1 & 207 & 52.3 & 0.52 & 317 & 7.06 & 14.4 & 1.96 & 124 \\
\hline$\hat{\mathrm{BQ}}$ & Deep gray mudstone & 35.9 & 4.51 & 124 & 15.2 & 44.8 & 37.4 & 102 & 250 & 51.2 & 0.5 & 389 & 6.39 & 18.3 & 2.35 & 119 \\
\hline $\mathrm{BQ}$ & Deep gray mudstone & 30.2 & 1.67 & 56.1 & 8.6 & 20 & 16.5 & 65.4 & 102 & 212 & 2.22 & 398 & 12.1 & 16 & 3.46 & 95.1 \\
\hline $\mathrm{BQ}$ & Deep gray mudstone & 58.8 & 4.14 & 106 & 16.7 & 43.8 & 27.1 & 58.1 & 214 & 94.8 & 0.5 & 655 & 17.9 & 15.2 & 3.55 & 108 \\
\hline $\mathrm{BQ}$ & Deep gray mudstone & 79.8 & 3.76 & 123 & 23.7 & 53.5 & 38 & 62.5 & 233 & 88.8 & 0.75 & 554 & 24.4 & 18.7 & 3.46 & 120 \\
\hline$\hat{\mathrm{BQ}}$ & Deep gray mudstone & 61.7 & 5.75 & 158 & 16.9 & 46.7 & 30.5 & 62.3 & 293 & 74.7 & 0.33 & 575 & 7.2 & 19 & 3.17 & 138 \\
\hline $\mathrm{BQ}$ & Deep gray mudstone & 36.9 & 1.68 & 49.8 & 8.8 & 24.5 & 46.2 & 65.1 & 129 & 201 & 0.61 & 555 & 16.6 & 15.5 & 2.71 & 105 \\
\hline $\mathrm{BQ}$ & Deep gray mudstone & 56.3 & 3.86 & 117 & 23.8 & 46.1 & 77.7 & 400 & 246 & 114 & 0.92 & 628 & 18.5 & 20.3 & 3.52 & 122 \\
\hline $\mathrm{BQ}$ & Deep gray mudstone & 57.8 & 3.82 & 125 & 17.7 & 34.7 & 22.7 & 55.8 & 222 & 145 & 0.77 & 599 & 14.9 & 20.7 & 3.15 & 135 \\
\hline$\hat{B Q}$ & Deep gray mudstone & 25.8 & 1.01 & 32.3 & 41.4 & 47.8 & 41.7 & 37.4 & 60.9 & 579 & 5.7 & 264 & 35.2 & 7.78 & 5.05 & 43.5 \\
\hline $\mathrm{BQ}$ & Deep gray mudstone & 59.1 & 3.72 & 120 & 15.7 & 34 & 30.9 & 49.7 & 230 & 96.2 & 0.76 & 678 & 18.8 & 20.6 & 3.42 & 107 \\
\hline$\hat{\mathrm{BQ}}$ & Deep gray mudstone & 52.5 & 3.4 & 103 & 15.9 & 35.7 & 29.4 & 55.6 & 211 & 111 & 0.97 & 793 & 31.1 & 21.9 & 5.12 & 147 \\
\hline $\mathrm{BQ}$ & Deep gray mudstone & 52.3 & 3.98 & 103 & 15.2 & 30.3 & 29 & 54.7 & 218 & 128 & 0.84 & 663 & 26.2 & 24.4 & 5.05 & 145 \\
\hline $\mathrm{BQ}$ & Deep gray mudstone & 49.4 & 2.57 & 95.8 & 16.5 & 32.6 & 33.3 & 50.5 & 151 & 200 & 0.87 & 619 & 33.2 & 26.8 & 5.09 & 169 \\
\hline $\mathrm{BQ}$ & Deep gray mudstone & 24.7 & 1.21 & 36.9 & 6.43 & 10.4 & 13.3 & 26.6 & 81.3 & 322 & 0.8 & 315 & 7.66 & 10.6 & 1.95 & 44.5 \\
\hline
\end{tabular}


Experiments were carried out in the State Key Laboratory of Oil and Gas Reservoir Geology and Exploitation, Chengdu University. The major elements were tested by X-ray fluorescence spectrometry (ZTIX-1)(XRF). The trace elements were analyzed by an inductively coupled plasma optical emission spectrometer (VISTA MPX) (ICP-OES) and an inductively coupled plasma mass spectrometer (X II) (ICP-MS). The TOC was detected by a carbon-sulfur analyzer (LECO CS230), and the carbon isotope of kerogen was tested by a stable isotope mass spectrometer (DELTA PLUS V). These experiments were conducted at $25{ }^{\circ} \mathrm{C}$. The experimental methods and instruments are in accordance with the National and Industry Standards.

\section{Paleoenvironment and Paleoproductivity}

\subsection{Paleoclimate}

The contents of the major and trace elements in sediments varied significantly under different climatic conditions, mainly affected by temperature and humidity. Therefore, chemical index alteration (CIA) would restore the paleoclimate (Equation (1)) [12].

$$
\mathrm{CIA}=\mathrm{Al}_{2} \mathrm{O}_{3} /\left(\mathrm{Al}_{2} \mathrm{O}_{3}+\mathrm{CaO}^{*}+\mathrm{Na}_{2} \mathrm{O}+\mathrm{K}_{2} \mathrm{O}\right) \times 100 \%
$$

where the content of elements is the mole fraction, and $\mathrm{CaO}^{*}$ represents $\mathrm{CaO}$ in silicate.

The CIA value of $80 \sim 100 \%$ reflects a hot and humid climate, 60 80\% reflects a warm and humid climate, and 50 60\% reflects a cold and dry climate $[13,14]$. The CIA value of the mudstones in the Meitan Formation is between $50.2 \%$ and $78.3 \%$, with an average of $69 \%$, showing a warm and humid climate as a whole (Figure 3a, Table 3).
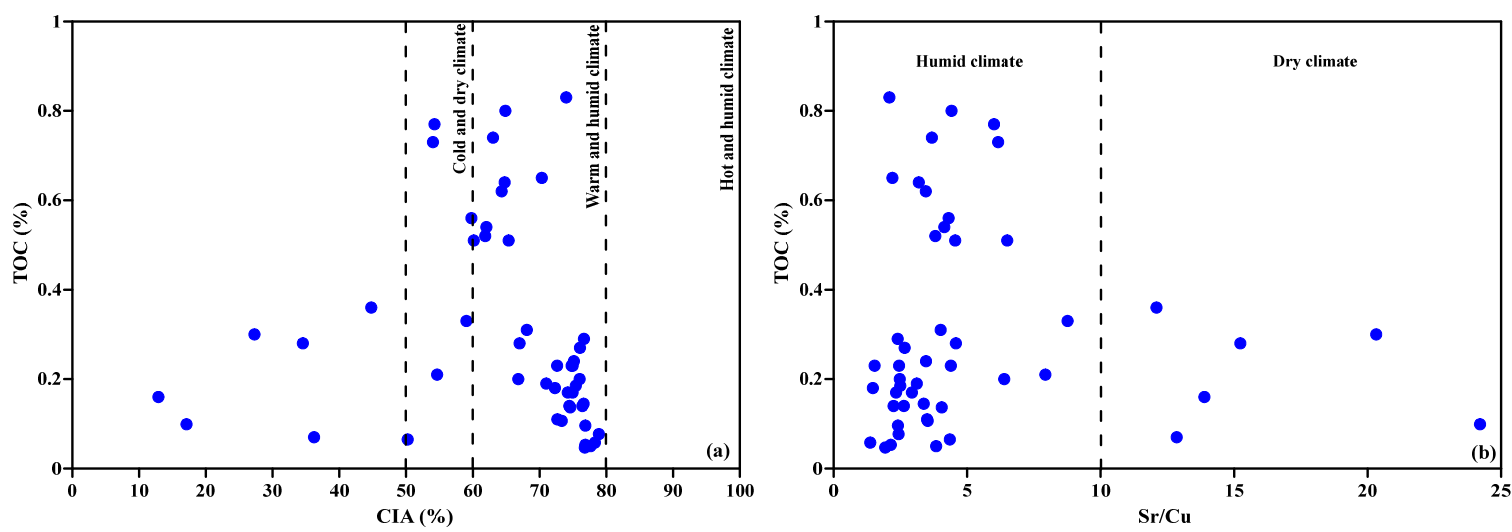

Figure 3. Paleoclimate discrimination of the mudstones in the Meitan Formation. TOC = Total organic carbon; CIA = Chemical index alteration. (a) relationship between CIA and TOC; (b) relationship between $\mathrm{Sr} / \mathrm{Cu}$ and TOC.

In addition, the $\mathrm{Sr} / \mathrm{Cu}$ ratio can also reflect the paleoclimate. Under a humid climate, the $\mathrm{Sr} / \mathrm{Cu}$ ratio is less than 10, and under a dry climate, the $\mathrm{Sr} / \mathrm{Cu}$ ratio is more than 10. The $\mathrm{Sr} / \mathrm{Cu}$ ratio of the mudstones in the Meitan Formation is between 1.34 and 24.2, with an average of 5.03, reflecting the humid climate (Figure 3b). Combined with the CIA and the $\mathrm{Sr} / \mathrm{Cu}$ ratio, the mudstones of the Meitan Formation were deposited under a warm and humid climate.

\subsection{Paleosalinity}

Paleosalinity is an important parameter reflecting the sea-level changes during geological histories. The $\mathrm{Rb} / \mathrm{K}$ ratio has a good positive correlation with paleosalinity, which can discriminate the paleosalinity. $\mathrm{Rb} / \mathrm{K}>0.006$ indicates the saline water deposition, $0.004<\mathrm{Rb} / \mathrm{K}<0.006$ indicates the brackish water deposition, and $\mathrm{Rb} / \mathrm{K}<0.004$ indicates freshwater deposition [15,16]. The $\mathrm{Rb} / \mathrm{K}$ ratio of mudstones in the Meitan Formation ranges from 0.0027 to 0.0069 , with an average of 0.0052 , indicating a brackish water sedimentary environment (Figure 4, Table 3). 
Table 3. Paleoenvironmental indicators of shale in the Meitan Formation.

\begin{tabular}{|c|c|c|c|c|c|c|c|c|c|c|c|}
\hline \multirow{2}{*}{$\begin{array}{l}\text { Well and Section } \\
\text { No. }\end{array}$} & \multirow{2}{*}{ Lithology } & \multicolumn{2}{|c|}{ Paleoclimate } & \multirow{2}{*}{$\begin{array}{c}\text { Paleosalinity } \\
\mathrm{Rb} / \mathrm{K}\end{array}$} & \multicolumn{4}{|c|}{ Paleoredox } & \multicolumn{3}{|c|}{ Paleoproductivity } \\
\hline & & CIA (\%) & $\mathrm{Sr} / \mathrm{Cu}$ & & $\mathrm{Th} / \mathrm{U}$ & $\mathrm{V} / \mathrm{Cr}$ & $\mathrm{V} /(\mathrm{V}+\mathrm{Ni})$ & $\mathrm{Ni} / \mathrm{Co}$ & $\mathrm{P} / \mathrm{Ti}$ & Baxs & Znxs \\
\hline JT1 & Gray calcareous mudstone & 34.53 & 15.23 & 0.0044 & 4.48 & 0.91 & 0.74 & 2.41 & 0.18 & 5604.67 & 29.87 \\
\hline JT1 & Gray calcareous mudstone & 27.28 & 20.32 & 0.0042 & 3.45 & 0.74 & 0.74 & 2.36 & 0.21 & 4649.00 & 32.90 \\
\hline JT1 & Gray calcareous mudstone & 44.78 & 12.09 & 0.0043 & 5.03 & 0.86 & 0.72 & 2.81 & 0.21 & $12,641.67$ & 47.37 \\
\hline JT1 & Gray calcareous mudstone & 59.04 & 8.76 & 0.0042 & 5.83 & 0.88 & 0.73 & 2.38 & 0.24 & 5956.00 & 70.00 \\
\hline JT1 & Gray calcareous mudstone & 60.14 & 6.50 & 0.0038 & 5.17 & 0.83 & 0.73 & 2.66 & 0.18 & $13,053.50$ & 28.40 \\
\hline WK1 & Grayish black mudstone & 73.32 & 3.52 & 0.0051 & 8.29 & 0.98 & 0.73 & 1.72 & 0.14 & 504.17 & 45.17 \\
\hline WK1 & Deep gray mudstone & 75.43 & 2.49 & 0.0050 & 7.62 & 0.79 & 0.75 & 2.51 & 0.14 & 494.33 & 66.33 \\
\hline WK1 & Deep gray mudstone & 76.41 & 2.24 & 0.0050 & 8.02 & 1.05 & 0.75 & 2.68 & 0.14 & 456.33 & 76.33 \\
\hline WK1 & Grayish black mudstone & 74.59 & 4.05 & 0.0050 & 8.05 & 1.00 & 0.74 & 1.98 & 0.17 & 440.00 & 72.00 \\
\hline QJ & Deep gray mudstone & 67.00 & 4.58 & 0.0059 & 6.72 & 1.14 & 0.72 & 2.33 & 0.24 & 143.50 & 77.50 \\
\hline $\mathrm{QJ}$ & Black mudstone & 68.09 & 4.01 & 0.0058 & 7.39 & 1.13 & 0.74 & 2.22 & 0.24 & 153.67 & 78.67 \\
\hline$\widehat{\mathrm{QJ}}$ & Shallow black mudstone & 75.14 & 3.46 & 0.0054 & 7.35 & 1.07 & 0.73 & 2.41 & 0.25 & 464.50 & 52.40 \\
\hline QJ & Dark black mudstone & 76.65 & 2.40 & 0.0055 & 7.49 & 1.26 & 0.75 & 2.97 & 0.11 & 366.50 & 45.50 \\
\hline $\mathrm{QJ}$ & Deep gray mudstone & 76.05 & 2.66 & 0.0055 & 7.67 & 1.15 & 0.73 & 2.77 & 0.15 & 386.00 & 31.40 \\
\hline QJ & Deep gray mudstone & 76.00 & 2.47 & 0.0052 & 7.65 & 1.12 & 0.77 & 3.45 & 0.13 & 524.00 & 30.90 \\
\hline $\mathrm{QJ}$ & Deep gray mudstone & 74.23 & 2.93 & 0.0049 & 6.42 & 1.10 & 0.75 & 2.97 & 0.20 & 730.33 & 29.63 \\
\hline$\widehat{\mathrm{QJ}}$ & Grayish black mudstone & 74.80 & 1.53 & 0.0048 & 7.21 & 1.34 & 0.75 & 3.95 & 0.13 & 882.83 & 45.63 \\
\hline$\widehat{W X T}$ & Dark black mudstone & 64.31 & 3.45 & 0.0064 & 5.70 & 1.61 & 0.78 & 2.72 & 0.11 & 316.33 & 58.63 \\
\hline WXT & Dark black mudstone & 64.77 & 3.19 & 0.0065 & 5.26 & 1.71 & 0.77 & 3.58 & 0.11 & 305.33 & 63.33 \\
\hline WXT & Dark black mudstone & 63.02 & 3.68 & 0.0065 & 5.21 & 1.51 & 0.75 & 2.71 & 0.11 & 306.33 & 66.33 \\
\hline WXT & Dark black mudstone & 59.78 & 4.31 & 0.0062 & 5.00 & 1.59 & 0.77 & 2.45 & 0.11 & 313.33 & 55.83 \\
\hline WXT & Dark black mudstone & 70.33 & 2.20 & 0.0064 & 5.22 & 1.95 & 0.77 & 3.80 & 0.09 & 526.17 & 84.17 \\
\hline WXT & Dark black mudstone & 73.99 & 2.09 & 0.0065 & 4.78 & 1.96 & 0.78 & 3.98 & 0.09 & 409.33 & 92.33 \\
\hline WXT & Dark black mudstone & 54.02 & 6.16 & 0.0066 & 4.10 & 1.60 & 0.73 & 3.04 & 0.10 & 680.50 & 72.50 \\
\hline WXT & Dark black mudstone & 61.85 & 3.81 & 0.0063 & 4.67 & 1.60 & 0.76 & 3.18 & 0.10 & 566.50 & 68.50 \\
\hline WXT & Dark black mudstone & 62.03 & 4.14 & 0.0067 & 4.86 & 1.78 & 0.77 & 2.78 & 0.10 & 746.83 & 77.83 \\
\hline WXT & Dark black mudstone & 65.36 & 4.55 & 0.0069 & 5.27 & 1.56 & 0.76 & 2.70 & 0.10 & 1781.00 & 73.00 \\
\hline PD & Yellow-green mudstone & 78.89 & 2.43 & 0.0026 & 8.67 & 1.14 & 0.73 & 1.84 & 0.14 & 203.00 & 89.00 \\
\hline PD & Yellow-green mudstone & 77.63 & 3.84 & 0.0055 & 8.62 & 1.18 & 0.76 & 2.85 & 0.11 & 146.67 & 59.77 \\
\hline PD & Gray-green mudstone & 76.85 & 2.14 & 0.0058 & 9.94 & 1.18 & 0.77 & 2.15 & 0.13 & 205.67 & 61.07 \\
\hline PD & Yellow-green mudstone & 76.86 & 2.41 & 0.0055 & 7.79 & 1.29 & 0.81 & 2.29 & 0.10 & 150.00 & 54.90 \\
\hline
\end{tabular}


Table 3. Cont.

\begin{tabular}{|c|c|c|c|c|c|c|c|c|c|c|c|}
\hline \multirow{2}{*}{$\begin{array}{l}\text { Well and Section } \\
\text { No. }\end{array}$} & \multirow{2}{*}{ Lithology } & \multicolumn{2}{|c|}{ Paleoclimate } & \multirow{2}{*}{$\begin{array}{c}\text { Paleosalinity } \\
\mathrm{Rb} / \mathrm{K}\end{array}$} & \multicolumn{4}{|c|}{ Paleoredox } & \multicolumn{3}{|c|}{ Paleoproductivity } \\
\hline & & CIA (\%) & $\mathrm{Sr} / \mathrm{Cu}$ & & $\mathrm{Th} / \mathrm{U}$ & $\mathrm{V} / \mathrm{Cr}$ & $\mathrm{V} /(\mathrm{V}+\mathrm{Ni})$ & $\mathrm{Ni} / \mathrm{Co}$ & $\mathrm{P} / \mathrm{Ti}$ & Baxs & Znxs \\
\hline $\mathrm{BQ}$ & Deep gray mudstone & 76.78 & 1.93 & 0.0042 & 7.35 & 1.01 & 0.74 & 3.32 & 0.13 & -181.33 & 52.77 \\
\hline $\mathrm{BQ}$ & Deep gray mudstone & 78.29 & 1.37 & 0.0054 & 7.79 & 1.04 & 0.73 & 2.95 & 0.13 & -131.00 & 62.00 \\
\hline $\mathrm{BQ}$ & Deep gray mudstone & 74.46 & 2.63 & 0.0043 & 6.36 & 1.03 & 0.75 & 2.17 & 0.17 & -141.17 & 34.93 \\
\hline $\mathrm{BQ}$ & Deep gray mudstone & 36.21 & 12.85 & 0.0048 & 4.62 & 0.59 & 0.74 & 2.33 & 0.17 & 8.00 & 35.40 \\
\hline $\mathrm{BQ}$ & Deep gray mudstone & 72.66 & 3.50 & 0.0047 & 4.28 & 0.98 & 0.71 & 2.62 & 0.17 & 80.83 & 13.93 \\
\hline $\mathrm{BQ}$ & Deep gray mudstone & 74.94 & 2.34 & 0.0050 & 5.40 & 1.03 & 0.70 & 2.26 & 0.12 & 12.33 & 20.83 \\
\hline $\mathrm{BQ}$ & Deep gray mudstone & 74.95 & 2.45 & 0.0052 & 5.99 & 1.14 & 0.77 & 2.76 & 0.10 & 33.33 & 20.63 \\
\hline $\mathrm{BQ}$ & Deep gray mudstone & 72.30 & 1.47 & 0.0051 & 5.77 & 0.96 & 0.72 & 1.94 & 0.18 & 151.33 & 363.33 \\
\hline $\mathrm{BQ}$ & Deep gray mudstone & 66.81 & 6.39 & 0.0050 & 6.57 & 0.93 & 0.78 & 1.96 & 0.19 & 79.00 & 15.80 \\
\hline $\mathrm{BQ}$ & Deep gray mudstone & 12.89 & 13.88 & 0.0053 & 1.54 & 0.74 & 0.40 & 1.15 & 5.43 & 112.33 & 25.73 \\
\hline $\mathrm{BQ}$ & Deep gray mudstone & 71.00 & 3.11 & 0.0050 & 6.02 & 1.12 & 0.78 & 2.17 & 0.15 & 114.67 & 6.37 \\
\hline $\mathrm{BQ}$ & Deep gray mudstone & 54.65 & 7.93 & 0.0042 & 6.64 & 0.59 & 0.76 & 1.91 & 0.38 & 107.33 & -9.87 \\
\hline $\mathrm{BQ}$ & Deep gray mudstone & 69.14 & 3.78 & 0.0046 & 4.28 & 0.70 & 0.74 & 2.25 & 0.36 & 132.17 & 4.77 \\
\hline $\mathrm{BQ}$ & Deep gray mudstone & 64.88 & 4.41 & 0.0048 & 4.83 & 0.71 & 0.77 & 1.99 & 0.41 & 23.83 & 5.53 \\
\hline $\mathrm{BQ}$ & Deep gray mudstone & 54.25 & 6.01 & 0.0042 & 5.27 & 0.57 & 0.75 & 1.98 & 0.52 & -52.67 & -1.17 \\
\hline $\mathrm{BQ}$ & Deep gray mudstone & 17.09 & 24.21 & 0.0051 & 5.44 & 0.83 & 0.78 & 1.62 & 0.16 & 109.17 & 10.77 \\
\hline
\end{tabular}




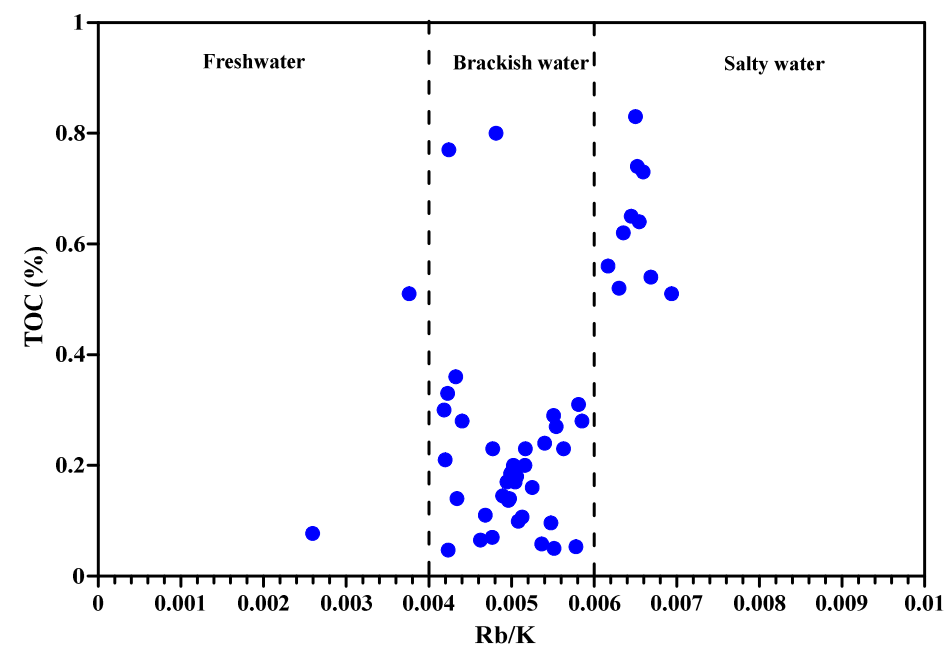

Figure 4. Paleosalinity discrimination of the mudstones in the Meitan Formation. TOC = Total organic carbon.

\subsection{Paleoredox}

Paleoredox has a significant influence on the preservation of organic matter. The redox conditions control the content of some major and trace elements, such as $\mathrm{V}, \mathrm{U}, \mathrm{Ni}$, and Th (Table 4). The Th/ $\mathrm{U}$ ratio and the $\mathrm{V} /(\mathrm{V}+\mathrm{Ni}$ ) ratio of the mudstones are 1.54 9.94 and 0.40 0.81, respectively, indicating the oxygen-depleted and anoxia environment (Figure $5 \mathrm{a}, \mathrm{b}$ ). However, the $\mathrm{V} / \mathrm{Cr}$ ratio and the $\mathrm{Ni} / \mathrm{Co}$ ratio are $0.47 \sim 1.96$ and 1.15 3.98, respectively, indicating the oxygen-enriched environment (Figure $5 \mathrm{c}, \mathrm{d}$, Table 3). Combined with the characteristic of black and gray-black mudstone in core and geological samples, the mudstones of the Meitan Formation are mainly deposited in an oxygen-poor and anoxic environment.

Table 4. Paleoredox evaluation index.

\begin{tabular}{ccccc}
\hline Index & Oxygen-Enriched & Oxygen-Depleted & Anoxia & References \\
\hline $\mathrm{Th} / \mathrm{U}$ & $>7.0$ & $2.0 \sim 7.0$ & $<2$ & {$[17]$} \\
$\mathrm{Ni} / \mathrm{Co}$ & $<5.0$ & $5.0 \sim 7.0$ & $>7.0$ & {$[12]$} \\
$\mathrm{V} / \mathrm{V}+\mathrm{Ni})$ & $<0.45$ & $0.45 \sim 0.60$ & $>0.60$ & {$[18]$} \\
$\mathrm{V} / \mathrm{Cr}$ & $<2.0$ & $2.0 \sim 4.25$ & $>4.25$ & {$[12]$} \\
\hline
\end{tabular}
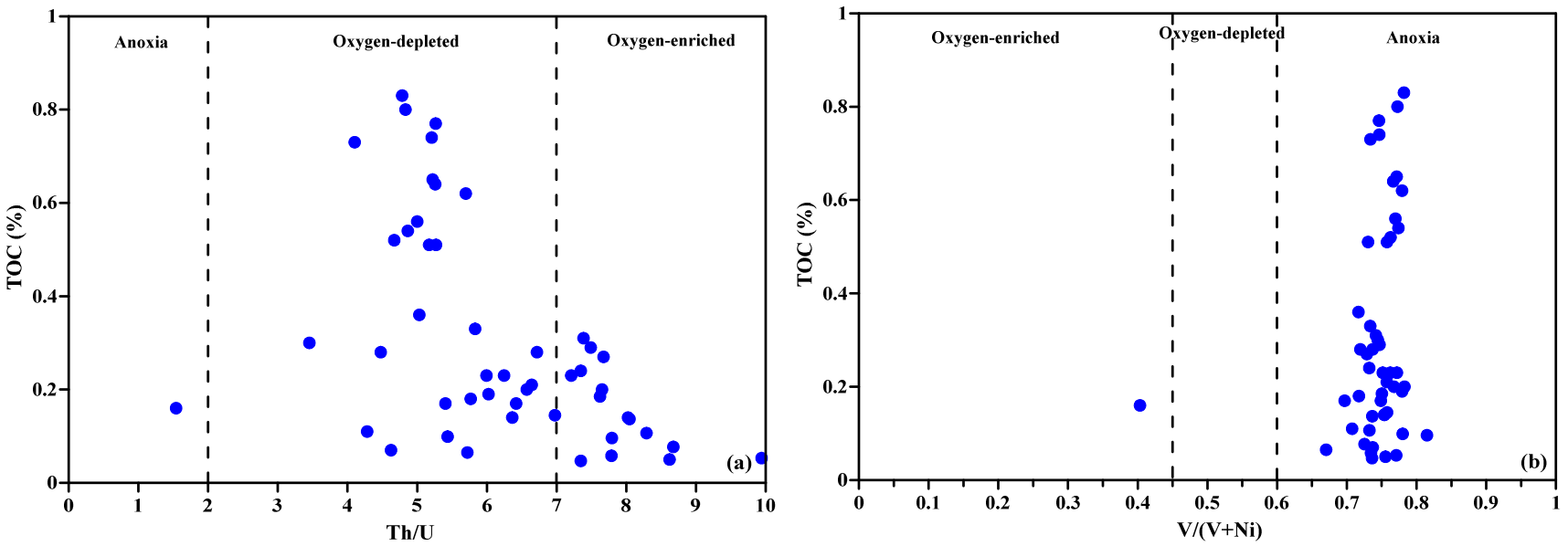

Figure 5. Cont. 

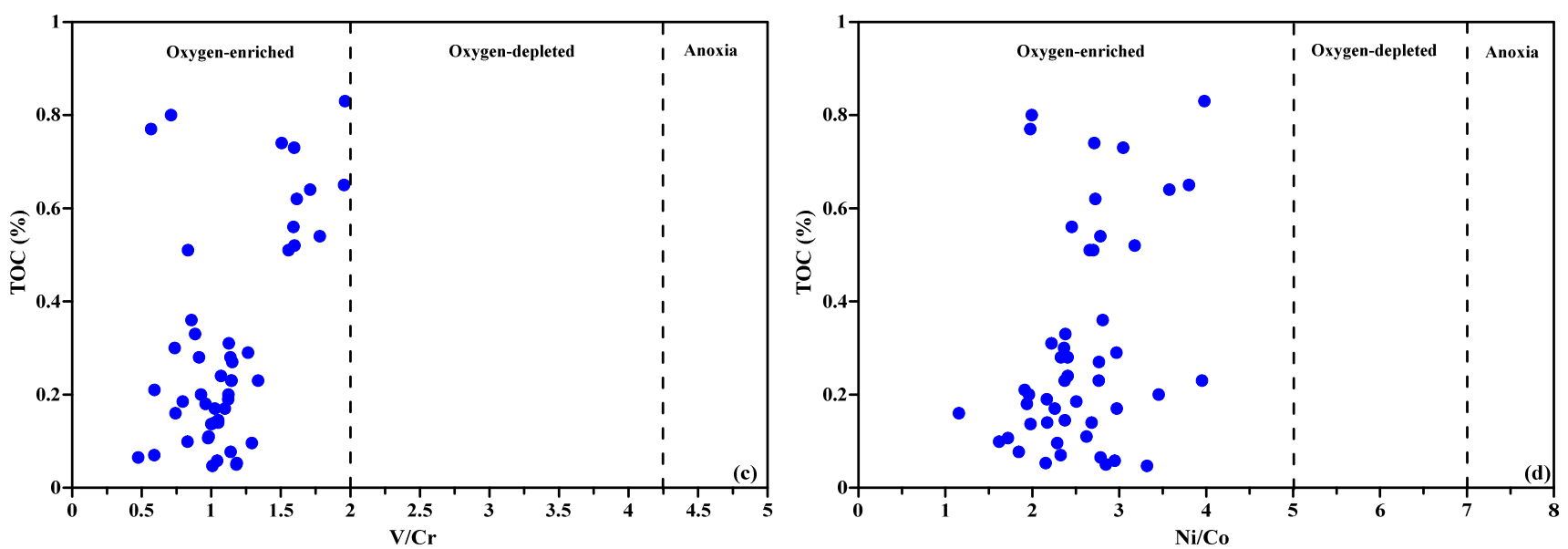

Figure 5. Paleoredox discrimination of the mudstones in the Meitan Formation. TOC $=$ Total organic carbon. (a) relationship between $\mathrm{Th} / \mathrm{U}$ and TOC; (b) relationship between $\mathrm{V} /(\mathrm{V}+\mathrm{Ni}$ ) and $\mathrm{TOC}$ (c): relationshio between $\mathrm{V} / \mathrm{Cr}$ and TOC (d): relationship between $\mathrm{Ni} / \mathrm{Co}$ and TOC.

\subsection{Paleoproductivity}

Paleoproductivity refers to the amount of organic carbon produced per time unit and volume unit of ancient marine organisms and is one of the controlling factors for source rocks $[19,20]$. The paleoproductivity can be qualitatively evaluated by major element $\mathrm{P}$ and trace elements $\mathrm{Ba}$ and $\mathrm{Zn}$, reflecting the marine paleoproductivity [21,22]. However, as terrestrial debris enters the ocean, it will cause deviations in the element content. Therefore, the element $\mathrm{Ti}$ is used to remove the influence of sedimentary organic matter and authigenic minerals (Equation (2)) [23,24].

$$
\mathrm{Xxs}=\mathrm{X}_{\text {total }}-\mathrm{Ti}_{\text {total }} \times(\mathrm{X} / \mathrm{Ti}) \mathrm{PAAS}
$$

where $X x s$ represents the corrected content of the element, $X_{\text {total }}$ represents the measured content of the element from samples, $\mathrm{Ti}_{\text {total }}$ represents the measured content of Ti from samples, and (X/Ti)PAAS is a constant, representing the ratio of the average content of $\mathrm{X}$ and $\mathrm{Ti}$ in the Neoarchean Australian shale. The (Ba/Ti)PAAS and (Zn/Ti)PAAS are 0.1083 and 0.0083 , respectively [25]. The corrected Xxs value is regular, indicating that the element in the sample is marine autogenic enrichment relative to PAAS, and negatively indicating that the element content is mainly contributed by terrestrial deposition [26]. The Baxs value of the mudstones in the Meitan Formation is between 8.0 and 13,053.5 ppm, with an average of $1227.4 \mathrm{ppm}$. The $\mathrm{Znxs}$ value is $4.8 \sim 363.3 \mathrm{ppm}$, with an average of 4233.7 ppm (Figure 6a,b, Table 3). The corrected Baxs and Znxs values indicate that the Meitan Formation has the characteristics of marine authigenic enrichment, and the higher element content indicated the higher productivity during the deposition period.

Moreover, the $\mathrm{P} / \mathrm{Ti}$ ratio can also indicate paleoproductivity. $\mathrm{P} / \mathrm{Ti}<0.34$ indicates low productivity, $0.34<\mathrm{P} / \mathrm{Ti}<0.79$ indicates medium productivity, and $\mathrm{P} / \mathrm{Ti}>0.79$ indicates high productivity. The P/Ti ratio of the mudstones in the Meitan Formation is between 0.09 and 0.52 , with an average of 0.28 , indicating that the Meitan Formation has low-medium productivity (Figure 6c, Table 3).

Combining the $\mathrm{P} / \mathrm{Ti}$ ratio, Baxs, and Znxs, the mudstones of the Meitan Formation have low-medium paleoproductivity. 

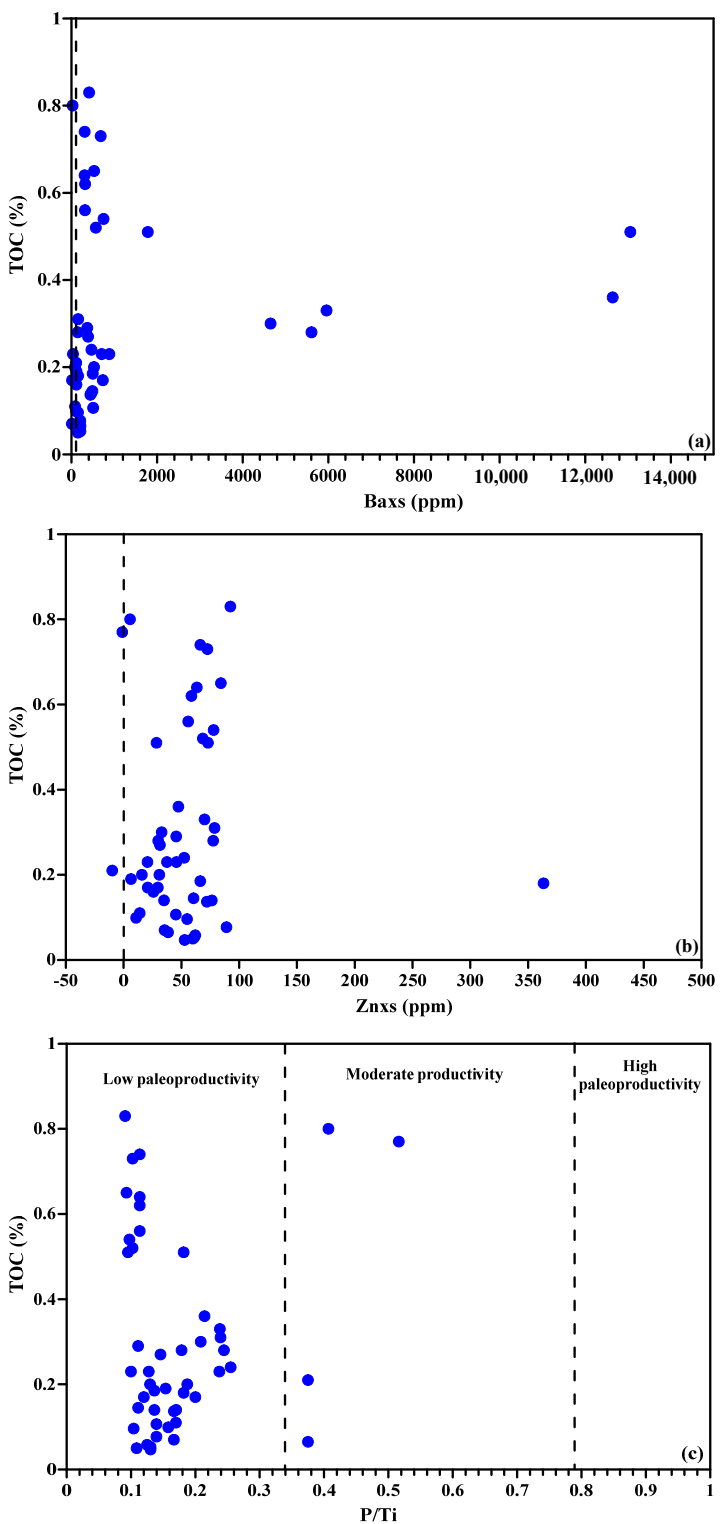

Figure 6. Paleoproductivity discrimination of the mudstones in the Meitan Formation. TOC = Total organic carbon. (a) relationship between Baxs and TOC; (b) relationship between Znxs and TOC; (c) relationship between $\mathrm{Pi} / \mathrm{Ti}$ and TOC.

\section{Discussion}

\subsection{Polysolution of the Paleoredox}

The restoration of paleoredox is the most important part of the paleoenvironment. The $\mathrm{Th} / \mathrm{U}, \mathrm{Ni} / \mathrm{Co}, \mathrm{V} /(\mathrm{V}+\mathrm{Ni})$, and $\mathrm{V} / \mathrm{Cr}$ ratios are the traditional redox indexes, in which $\mathrm{Th} / \mathrm{U}$ and $\mathrm{V} /(\mathrm{V}+\mathrm{Ni})$ of the Meitan Formation indicate the oxygen-depleted and anoxic environment, while $\mathrm{V} / \mathrm{Cr}$ and $\mathrm{Ni} / \mathrm{Co}$ indicate the oxygen-enriched environment.

However, researchers have realized the limitations of element ratios to evaluate the paleoredox [27-29]. The threshold values corresponding to the same index are not uniform. For example, the threshold of U/Th to evaluate the paleoredox proposed by Jones and Manning [12] and Wignall and Hallam [17] are quite different. This is because the geological conditions of different research areas, such as geological ages, provenance, and paleoactivities, are quite different, and these thresholds do not have global applicability. Except for the redox environment, the organic matter types, depositional rate, and late diagenesis may affect the enrichment of trace elements. Thus, these indexes have multiple conclusions for the determination of redox. Therefore, trace element discrimination can 
only be used as a reference in the recovery of paleoredox, but also combined with the actual lithology and color of samples. In 50 mudstone samples, $90 \%$ of the samples are dark gray and black mudstones, indicating the oxygen-depleted and anoxic environments. Combined with the sample color and trace element ratios, the mudstones of the Meitan Formation were deposited under an oxygen-depleted and anoxic environment.

\subsection{Control of Paleoenvironment on Source Rock Development}

Paleoproductivity and preservation conditions are the main factors controlling the enrichment of organic matters [30-32].

Two shaly horizons at the Upper Ordovician Fjäcka and Mossen Formations are rich in organic matters and are one of the main source rocks in the central part of the Baltic Basin, Southwestern Lithuania. They were recognized as being formed in oxygen-depleted benthic settings, as indicated by their high TOC, with the average value of $3.28 \%$. The source rocks were deposited in marine, non-carbonate settings and the paleoproductivity was high [33]. Ordovician black mudstones are the fair source rocks in the Paleozoic petroleum system in Iraq, and their average TOC is $0.9 \%$. The source rocks were developed in a marine environment, the paleoredox indicators prove the oxygen-depleted and oxygenenriched conditions, and the CIA is low, with the value of $52 \% \sim 58 \%$ [34]. Dark mudstones of the Ordovician Tanjianshan Formation of the Qaidam Basin, China, are high-quality source rocks with a high hydrocarbon-production potential. The average TOC of the source rocks is $1.75 \%$, the organic matter has reached a mature-overmature stage, and the kerogen was mainly typeII ${ }_{1}$. The major and trace elements indicate that the source rocks were deposited in a paleoenvironment with restricted water and paleoredox conditions, which played a significant role in the organic matter enrichment [35]. Compared with the paleoenvironment and the quality of the Ordovician source rocks in the above three basins, the quality of the Meitan Formation can be evaluated. The mudstones of the Meitan Formation were deposited in an oxygen-depleted and anoxic environment of brackish water. The retention environment of the anoxic bottom water and the slow deposition rate were beneficial to the preservation of organic matter in sedimentary rocks [36,37]. Meantime, a warm and humid climate was conducive to the reproduction of organisms during the depositional period of the Meitan Formation, with low-medium paleoproductivity. In general, the paleoenvironment is conducive to the development of poor and moderate source rocks.

The TOC content of the mudstone in the Meitan Formation is $0.03 \% \sim 2.34 \%$, with an average of $0.43 \%$ (Table 5 and Table S1). The source rocks only developed in the northern, western, and southern blocks, and the TOC content is $0.40 \sim 2.34 \%$, with an average of $0.78 \%$. According to the classification standard for Paleozoic marine source rocks [38], the evaluations of the source rocks of the northern and southern blocks are poor and moderate, and the ones in the western block are poor (Figure 1), which is consistent with the paleoenvironmental restoration. The kerogen carbon isotope of the Meitan Formation is between $-31.99 \%$ and $-26.6 \%$, with an average of $-29.32 \%$, which has the characteristics of type $\mathrm{II}_{1}$ kerogen and the potential of generating oil and gas (Figure 7).

Table 5. Total organic carbon content of mudstone in the Meitan Formation.

\begin{tabular}{|c|c|c|c|c|c|c|c|c|c|}
\hline Well No. & $\begin{array}{c}\text { TOC } \\
(\%)\end{array}$ & Well No. & $\begin{array}{c}\text { TOC } \\
(\%)\end{array}$ & Well No. & $\begin{array}{c}\text { TOC } \\
(\%)\end{array}$ & Well No. & $\begin{array}{c}\text { TOC } \\
(\%)\end{array}$ & Well No. & $\begin{array}{c}\text { TOC } \\
(\%)\end{array}$ \\
\hline JT1 & $\frac{0.27-0.51}{0.35(14)}$ & LL1 & $\frac{0.10-0.61}{0.29(9)}$ & $\mathrm{Z3}$ & \multirow{6}{*}{$\begin{array}{c}\frac{0.15}{0.15(1)} \\
\frac{0.12-0.46}{0.21(6)} \\
\frac{0.13-0.84}{0.30(16)} \\
\frac{0.14-1.04}{0.40(19)} \\
\underline{0.08-0.42} \\
\frac{0.21(19)}{0.08-0.25} \\
0.15(20)\end{array}$} & YS1 & \multirow{6}{*}{$\begin{array}{l}\frac{0.03-0.09}{0.06(9)} \\
\frac{0.03-0.25}{0.10(37)} \\
\frac{0.30-0.74}{0.47(8)} \\
\frac{0.22-1.59}{0.49(18)} \\
\frac{0.31-0.74}{0.50(36)} \\
\frac{0.17-0.87}{0.51(13)}\end{array}$} & QJ & \multirow{6}{*}{$\begin{array}{l}\frac{0.14-0.31}{0.22(22)} \\
\frac{0.51-0.88}{0.66(20)} \\
\frac{0.049-0.17}{0.08(15)} \\
\frac{0.08-0.37}{0.18(12)} \\
\frac{0.17-0.84}{0.50(16)} \\
\frac{0.76-1.54}{1.17(9)} \\
\end{array}$} \\
\hline MS1 & $\frac{0.41-2.34}{1.07(59)}$ & WK1 & $\frac{0.04-0.20}{0.14(9)}$ & $\mathrm{C} 7$ & & YS2 & & WXT & \\
\hline TT1 & $\frac{0.13-0.63}{0.28(33)}$ & L32 & $\frac{0.07-0.48}{0.22(11)}$ & W2 & & GT2 & & PD & \\
\hline LS1 & $\frac{0.23-0.49}{0.41(7)}$ & LT1 & $\frac{0.29-0.60}{0.45(8)}$ & W15 & & HS1 & & LZY & \\
\hline ZS1 & $\frac{0.21-0.69}{0.36(9)}$ & WS1 & $\frac{0.11-2.29}{0.36(14)}$ & WH103 & & $\mathrm{NC} 1$ & & SQ & \\
\hline A8 & $\frac{0.16-0.28}{0.21(6)}$ & G2 & $\frac{0.18-0.61}{0.37(11)}$ & WH104 & & $\mathrm{HT}$ & & HHY & \\
\hline
\end{tabular}




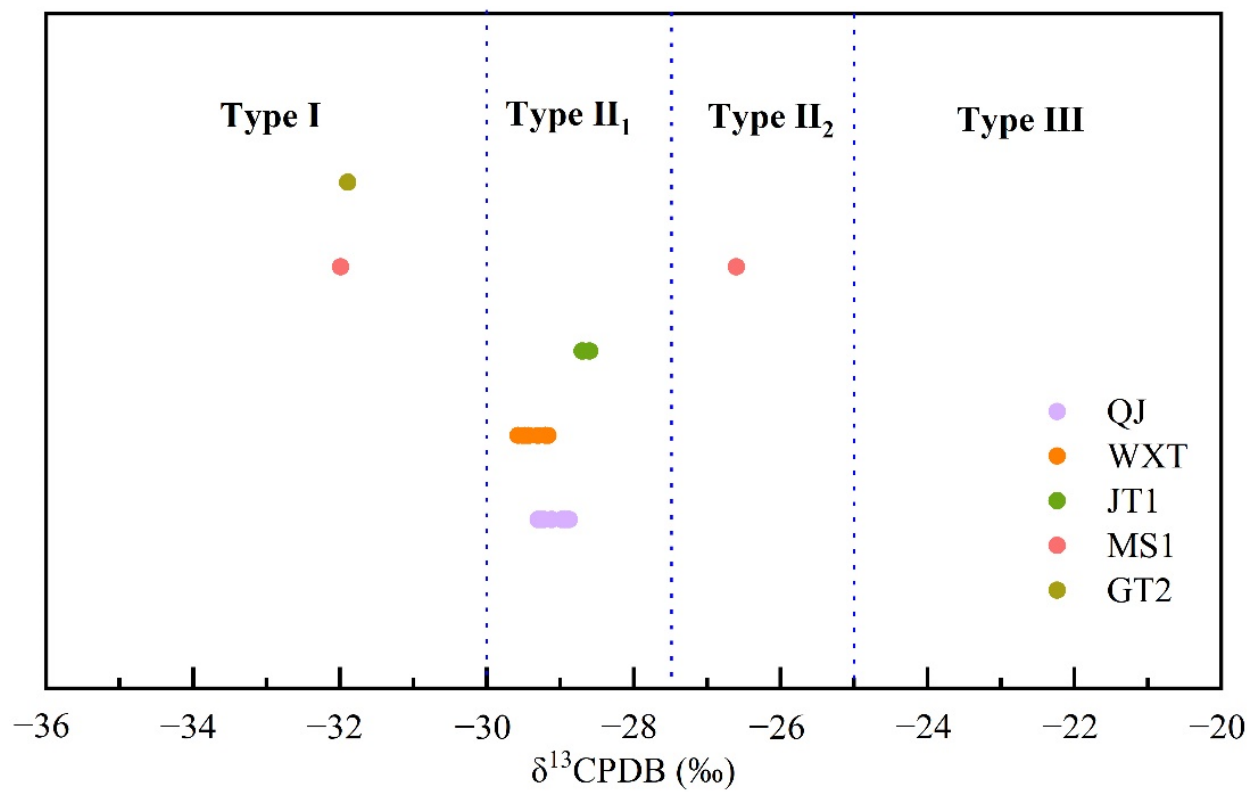

Figure 7. Kerogen types of source rocks in the Meitan Formation (Kerogen type classification is based on [39]).

In summary, the source rocks of the Meitan Formation are only developed in three blocks and have hydrocarbon generation potential. Therefore, the source rocks and adjacent reservoirs of the Meitan Formation can be the concurrent layers during the oil and gas exploration.

\section{Conclusions}

(1) The mudstones of the Meitan Formation were deposited in an oxygen-depleted and anoxic environment of brackish water. The paleoclimate is warm and humid, and the paleoproductivity is low to medium, which is beneficial to the development of poor and moderate source rocks.

(2) Controlled by the paleoenvironment, the kerogen type of the source rocks in the Meitan Formation is mainly type $\mathrm{II}_{1}$. Poor and moderate source rocks were developed in the northern and southern blocks, and only poor source rocks were developed in the western block.

(3) In general, the source rocks of the Meitan Formation have hydrocarbon generation potential, and oil and gas can migrate and accumulate in the upper reservoirs. The Meitan Formation and its adjacent reservoirs can be de concurrent layers during the petroleum exploration in the Ordovician. In the next Ordovician petroleum exploration, gas and source rock correlation in the upper Baota Formation can be considered to reveal whether there is a contribution of the Meitan Formation. In addition, for the source rocks deposited in a similar paleoenvironment, the possibility of small-scaled oil and gas discovery can be considered.

Supplementary Materials: The following supporting information can be downloaded at: https: / / www.mdpi.com/article/10.3390/min12010075/s1, Table S1: Total organic carbon content (TOC) of mudstone in the Meitan Formation.

Author Contributions: Conceptualization, M.Y., Y.Z. and X.F.; methodology, M.Y. and Y.Z.; experiment, M.Y. and L.Q.; writing—original draft, M.Y.; writing—review and editing, Y.Z., X.F. and W.L.; funding acquisition, X.F. W.L. and J.Z. (Jianyong Zhang); language and grammar revision, Z.Z. and J.Z. (Jiazhen Zhang). All authors have read and agreed to the published version of the manuscript.

Funding: This research was funded by the Key Technology Projects of PetroChina Natural Gas Co., Ltd. (No. 2018A-0105) and the National Natural Science Foundation of China (No. 41772248). 
Data Availability Statement: Data are contained in the tables of the article.

Acknowledgments: The authors thank the PetroChina Hangzhou Research Institute of Geology for their strong support in data use and funding. The authors also thank the reviewers for their constructive suggestions on the modification and improvement of the manuscript.

Conflicts of Interest: The authors declare no conflict of interest.

\section{References}

1. Zuo, Y.H.; Jiang, S.; Wu, S.H.; Xu, W.; Zhang, J.; Feng, R.P.; Yang, M.H.; Zhou, Y.S.; Santosh, M. Terrestrial heat flow and lithospheric thermal structure in the Chagan Depression of the Yingen-Ejinaqi Basin, north central China. Basin Res. 2020, 32, 1328-1346. [CrossRef]

2. El Atfy, H.; Ghassal, B.I.; Maher, A.; Hosny, A.; Mostafa, A.; Littke, R. Palynological and organic geochemical studies of the Upper Jurassic-Lower Cretaceous successions, Western Desert, Egypt: Implications for paleoenvironment and hydrocarbon source rock potential. Int. J. Coal Geol. 2019, 211, 103207. [CrossRef]

3. Enciso-Cárdenas, J.J.; Núñez-Useche, F.; Ortegon, L.F.C.; de la Rosa-Rodríguez, G.; Martínez-Yañez, M.; Borrego, Á.G. Paleoenvironment and source-rock potential of the Cenomanian-Turonian Eagle Ford Formation in the Sabinas basin, northeast Mexico. J. S. Am. Earth Sci. 2021, 108, 103184. [CrossRef]

4. Guo, T.L. Charateristics and exploration potential of Ordovician reservoirs in Sichuan Basin. Oil Gas Geol. 2014, 35, 372-378, (In Chinese with English Abstract).

5. Huang, W.M.; Liu, S.G.; Ma, W.X.; Wang, G.Z.; Zhang, C.J.; Zeng, X.L. Petroleum exploration potential of the Ordovician in the Sichuan Basin. Oil Gas Geol. 2011, 3, 461-473, (In Chinese with English Abstract).

6. Zhang, D.W.; He, Z.L.; Li, G.L. Geochemistry and accumulation model of Ordovician hydrocarbon in Sichuan Basin. Nat. Gas Geosci. 2020, 31, 428-435. [CrossRef]

7. Zhang, D.W.; Hao, Y.Q.; Zhang, R.Q.; Sun, W.; Gao, P.; Li, G.L. Hydrocarbon potential analysis and exploration significance of the Meitan Formation, Sichuan Basin. Acta Sedimentol. Sin. 2020, 38, 635-647.

8. Liu, S.G.; Yang, Y.; Deng, B.; Zhong, Y.; Wen, L.; Sun, W.; Li, Z.W.; Jansa, L.; Li, J.X.; Song, J.M.; et al. Tectonic evolution of the Sichuan Basin, Southwest China. Earth-Sci. Rev. 2021, 213, 103470. [CrossRef]

9. He, D.F.; Lu, R.Q.; Huang, H.Y.; Wang, X.S.; Jiang, H.; Zhang, W.K. Tectonic and geological setting of the earthquake hazards in the Changning shale gas development zone, Sichuan Basin, SW China. Pet. Explor. Dev. 2019, 46, 1051-1064. [CrossRef]

10. Li, J. Ordovician acritarchs from the Meitan Formation of Guizhou Province, sourhwest China. Palaeontology 1987, 30, 613-634.

11. He, Y.Y.; Niu, Z.J.; Zhang, Z.Z.; Song, F.; Yao, H.Z.; Yang, W.Q.; Gong, Y.J.; Wang, Z.W.; Gan, J.M.; Wang, Z.H. Detrital zircons of the Meitan Formation during Ordovician in Northeastern Guizhou and its significance for provenance-tectonic and implications for metallogenic chronology. Geol. China 2020, 47, 1025-1040, (In Chinese with English Abstract).

12. Jones, B.; Manning, D.A.C. Comparison of geochemical indices used for the interpretation of paleoredox conditions in ancient mudstones. Chem. Geol. 1994, 111, 111-129. [CrossRef]

13. Nesbitt, H.W.; Young, G.M. Early Proterozoic climates and plate motions inferred from major element chemistry of lutites. Nature 1982, 299, 715-717. [CrossRef]

14. Kasanzu, C.H.; Maboko, M.A.H.; Manya, S. Reconstruction of Pliocene-Pleistocene sediment sources and weathering intensity in the paleo-life rich Olduvai and Laetoli basins of northern Tanzania using major and trace element geochemistry and Sr isotopic data. J. Afr. Earth Sci. 2016, 123, 89-98. [CrossRef]

15. Wang, Y.Y.; Guo, W.Y.; Zhang, G.D. Application of some geochemical indicators in determining of sedimentary environment of the Funing Group(Paleogene), Jin-Hu Depression, Kiangsu Province. J. Tongji Univ. 1979, 7, 51-60, (In Chinese with English Abstract).

16. Chen, P.; Lin, W.B.; Gong, D.J.; Shang, F.; Liu, X.T. Sedimentary geochemical characteristics and its sedimentary environment significance of the black shale of the Lower Cambrian Bianmachong Formation in the Cen'gong block, Guizhou Province. Chin. Chin. J. Geol. 2020, 55, 1025-1043.

17. Wignall, P.B.; Hallam, A. Anoxia as a cause of the Permian/Triassic mass extinction: Facies evidence from northern Italy and the western United States. Palaeogeogr. Palaeoclim. Palaeoecol. 1992, 93, 21-46. [CrossRef]

18. Hatch, J.R.; Leventhal, J.S. Relationship between inferred redox potential of the depositional environment and geochemistry of the Upper Pennsylvanian (Missourian) Stark Shale Member of the Dennis Limestone, Wabaunsee County, Kansas, U.S.A. Chem. Geol. 1992, 99, 65-82. [CrossRef]

19. Arthur, M.A.; Sageman, B.B. Marine Black Shales: Depositional Mechanisms and Environments of Ancient Deposits. Annu. Rev. Earth Planet. Sci. 1994, 22, 499-551. [CrossRef]

20. Tribovillard, N.; Algeo, T.J.; Lyons, T.; Riboulleau, A. Trace metals as paleoredox and paleoproductivity proxies: An update. Chem. Geol. 2016, 232, 12-32. [CrossRef]

21. Brumsack, H.-J. The trace metal content of recent organic carbon-rich sediments: Implications for Cretaceous black shale formation. Palaeogeogr. Palaeoclimatol. Palaeoecol. 2006, 232, 344-361. [CrossRef] 
22. Han, S.B.; Zhang, Y.L.; Huang, J.; Rui, Y.R.; Tang, Z.Y. Elemental Geochemical Characterization of Sedimentary Conditions and Organic Matter Enrichment for Lower Cambrian Shale Formations in Northern Guizhou, South China. Minerals 2020, 10, 793. [CrossRef]

23. Yan, D.T.; Chen, D.Z.; Wang, Q.C.; Wang, J.G. Predominance of stratified anoxic Yangtze Sea interrupted by short-term oxygenation during the Ordo-Silurian transition. Chem. Geol. 2012, 291, 69-78. [CrossRef]

24. Algeo, T.J.; Kuwahara, K.; Sano, H.; Bates, S.; Lyons, T.; Elswick, E.; Hinnov, L.; Ellwood, B.; Moser, J.; Maynard, J.B. Spatial variation in sediment fluxes, redox conditions, and productivity in the Permian-Triassic Panthalassic Ocean. Palaeogeogr. Palaeoclimatol. Palaeoecol. 2011, 308, 65-83. [CrossRef]

25. Taylor, S.R.; McLennan, S.M. The Continental Crust: Its Composition and Evolution; Blackwell Malden Mass: Hoboken, NJ, USA, 1985.

26. Wang, Z.X.; Lü, X.X.; Qian, W.W. Geochemical characteristics of the Cambrian marine carbonate elements and its petroleum geological significance: Case study of Xiaoerbulake Formation in Keping area of Tairm Basin. Nat. Gas Geosci. 2017, 28, 1085-1095. [CrossRef]

27. Rimmer, S.M.; Thompson, J.A.; Goodnight, S.A.; Robl, T. Multiple controls on the preservation of organic matter in DevonianMississippian marine black shales: Geochemical and petrographic evidence. Palaeogeogr. Palaeoclimatol. Palaeoecol. 2004, 215, 125-154. [CrossRef]

28. Xu, G.; Hannah, J.L.; Bingen, B.; Georgiev, S.L.; Stein, H.J. Digestion methods for trace element measurements in shales: Paleoredox proxies examined. Chem. Geol. 2012, 324, 132-147. [CrossRef]

29. Liang, H.R.; Xu, G.S.; Xu, F.H.; Yu, Q.; Liang, J.J.; Wang, D.Y. Paleoenvironmental evolution and organic matter accumulation in an oxygen-enriched lacustrine basin: A case study from the Laizhou Bay Sag, southern Bohai Sea (China). Int. J. Coal Geol. 2020, 217, 103318. [CrossRef]

30. Ramanampisoa, L. Primary control of paleoproduction on organic matter preservation and accumulation in the Kimmeridge rocks of Yorkshire (UK). Org. Geochem. 1994, 21, 1153-1167. [CrossRef]

31. Hladyniuk, R.; Longstaffe, F.J. Paleoproductivity and organic matter sources in Late Quaternary Lake Ontario. Palaeogeogr. Palaeoclimatol. Palaeoecol. 2015, 435, 13-23. [CrossRef]

32. Overare, B.; Osokpor, J.; Ekeh, P.C.; Azmy, K. Demystifying provenance signatures and paleo-depositional environment of mudrocks in parts of south-eastern Nigeria: Constraints from geochemistry. J. Afr. Earth Sci. 2020, 172, 103954. [CrossRef]

33. Cichon-Pupienis, A.; Littke, R.; Froidl, F.; Lazauskienė, J. Depositional history, source rock quality and thermal maturity of Upper Ordovician - Lower Silurian organic-rich sedimentary rocks in the central part of the Baltic Basin. Mar. Pet. Geol. 2020, 112, 104083. [CrossRef]

34. Mohammed, I.Q.; Farouk, S.; Baioumy, H.; Lotfy, N.M.; Al-Hadidy, A.H. Mineralogical and geochemical characteristics of the Paleozoic source rocks, Akkas gas field, Western Desert of Iraq: Implications for their origin, maturation and Ordovician-Silurian transition. Mar. Pet. Geol. 2020, 118, 104432. [CrossRef]

35. Wu, Y.P.; Liu, C.L.; Liu, Y.J.; Gong, H.W.; Awan, R.S.; Li, G.X.; Zang, Q.B. Geochemical characteristics and the organic matter enrichment of the Upper Ordovician Tanjianshan Group, Qaidam Basin, China. J. Pet. Sci. Eng. 2022, 208a, 109383. [CrossRef]

36. Wani, H.; Mondal, M. Geochemical characteristics of calcareous and non-calcareous shales from the Meso-Neoproterozoic basins of the Bastar craton, Central Indian Shield: Implications for provenance, tectonic setting, paleoweathering and paleoredox conditions. Geosci. J. 2011, 15, 275-286. [CrossRef]

37. Awan, R.S.; Liu, C.; Gong, H.; Dun, C.; Tong, C.; Chamssidini, L.G. Paleo-sedimentary environment in relation to enrichment of organic matter of Early Cambrian black rocks of Niutitang Formation from Xiangxi area China. Mar. Pet. Geol. 2020, $112,104057$. [CrossRef]

38. Liang, D.G.; Guo, T.L.; Chen, J.P.; Bian, L.Z.; Zhao, Z. Some progresses on studies of hydrocarbon generation and accumulation in marine sedimentary regions, southern China (Part 1): Distribution of four suits of regional marine source rocks. Mar. Orig. Pet. Geol. 2008, 2, 1-16.

39. Li, G.J.; Xia, G.Q.; Yi, H.S.; Ji, C.J.; Yang, J.B. Characteristics of the Mesozoic marine argillaceous source rocks and prediction of favorable hydrocarbon generation area in South Qiangtang Depression, Xizang (Tibet). Geol. Rev. 2020, 66, 1241-1260. 